\title{
Which Comes First: Increased Intestinal Paracellular Permeability or Subepithelial Inflammation?
}

\author{
Yasutada Akiba ${ }^{1}$ \\ Accepted: 16 November 2020 / Published online: 6 January 2021 \\ ○) Springer Science+Business Media, LLC, part of Springer Nature 2021
}

The intestinal barrier is the sum of multiple mechanisms, including those that regulate transepithelial electrical resistance, the transepithelial movement of small molecules, macromolecules, and particles, mucus secretion, and the bidirectional transport of water and ions. A key component of the intestinal barrier is paracellular permeability to solutes that traverse intercellular tight junctions [1]. Increased intestinal paracellular permeability is linked to the metabolic syndrome, obesity, diabetes, and fatty liver, in which chronic low-grade inflammation of adipose tissue, the pancreatic islets, and the liver is associated with measurable elevations of circulating lipopolysaccharides (LPS), termed metabolic endotoxemia, associated with enhanced LPS entry from the intestinal lumen into the circulation possibly via increased intestinal paracellular permeability [2]. Intestinal paracellular permeability is primarily regulated by intrinsic tight junction (TJ) proteins, including zonula occludens-1 (ZO-1), the occludins, and the claudins [3]. Changes in the expression of TJ proteins and their structure are well studied and correlate with paracellular permeability to molecular size markers such as FITC-dextran, polyethylene glycol, and carbohydrates and polyols such as mannitol and lactulose [4]. Although there is an extensive literature documenting associations among TJ protein abundance and structure, epithelial barrier function, and numerous disease states, a causeand-effect relationship among these factors and the time course of barrier dysfunction changes have not been fully evaluated. Furthermore, there is often confusion regarding the contributions of individual intestinal segments toward the regulation of overall gut permeability. For instance, it is not known which specific intestinal segment is responsible

Yasutada Akiba

yakiba@mednet.ucla.edu

1 Veterans Affair Greater Los Angeles Healthcare System and Department of Medicine, David Geffen School of Medicine, UCLA, B114, R217, West Los Angeles VAMC, 11301 Wilshire Blvd., Los Angeles, CA 90073, USA for the paracellular absorption of macromolecular solutes, and if paracellular permeability changes precede or follow the development of metabolic syndrome. High-fat dietinduced elevation in circulating lipopolysaccharides (LPS) levels are explained by increased paracellular permeability, but in which intestinal segment? Although the density of Gram-negative microbes and thus LPS concentrations in the cecum or colon can be a millionfold or more greater than in the jejunum, it appears that in some instances such as in coprophagic rodents, the proximal small intestinal lumen also has high luminal LPS levels [5], suggesting that segmental differences should be examined in order to determine the segments responsible for absorption of specific substances.

Other issues regarding the interpretation of intestinal permeability measurements include whether transport is via paracellular diffusion versus transcellular transport, and the timing of permeability changes, that can be classified as: acute $(<30 \mathrm{~min} \sim 1 \mathrm{~h}$ ), subacute (3-24 h), longer duration ( $\sim 1$ week), or chronic ( $>30$ days). For instance, systemic inflammation such as endotoxemia increases small intestinal paracellular permeability to FITC-dextran 4000 (FD4) via the paracellular leak pathway within $3 \mathrm{~h}$, changes that continue for at least $48 \mathrm{~h}$ in rats [6]. In contrast, in normal jejunum, data suggest that LPS are transported via transcellular pathways into the portal vein within 15-30 min and lymphatic circulation at $60-90 \mathrm{~min}$ following luminal exposure to long-chain fatty acid oleic acid in vivo and in vitro without corresponding changes in paracellular permeability [5], suggesting that proinflammatory bacterial components may physiologically cross the small intestinal epithelium. Therefore, chronically absorbed LPS or other inflammatory factors may trigger low-grade inflammation during high-fat diet consumption causing intestinal subepithelial inflammation, followed by increased paracellular permeability linked to metabolic endotoxemia, fatty liver, obesity and diabetes.

In this issue of Digestive Diseases and Sciences, Nascimento al. [7] hypothesize that a high-fat diet impairs 
proximal small intestinal barrier function prior to the development of type 2 diabetes with metabolic endotoxemia, based on their former study that demonstrated changes in tight junction morphology and paracellular permeability to FITC-dextran in the ileum and colon do not contribute to the onset of type 2 diabetes [8]. They found that the expression of claudins-1, -2 , and -3 and $\mathrm{ZO}-1$ in duodenal and jejunal epithelial cells as measured by immunofluorescence were decreased as early as 15 days after the initiation of a highfat diet, prior to the development of hyperglycemia, which occurred after 30 days, followed by increased intestinal permeability to the small molecule Lucifer yellow at 60 days. Nevertheless, these changes were not associated with changes in total immunoreactive tissue TJ protein amount, plasma LPS and zonulin, or tissue TNF- $\alpha$ abundance. These data suggest that a high-fat diet increases intestinal small molecule paracellular permeability by derangement of TJ structure in the proximal small intestine, rather than in the distal small intestine or colon, preceding the onset of type 2 diabetes. This is an informative study providing the responsible intestinal segments and a step-by-step mechanism of a high-fat diet-induced onset of type 2 diabetes. It is still unclear what specifically affects TJ protein structure, whether TJ structural changes in the duodenum and jejunum directly contribute to the increased paracellular permeability to Lucifer yellow, and when metabolic endotoxemia and low-grade inflammation are triggered.

This is a classic 'chicken or egg' situation in that it is unclear which comes first, increased paracellular permeability or inflammation. Luminal nutrients, bacterial metabolites, or toxins that are affected by a high-fat diet through fatinduced dysbiosis of the gut microbiome may alter intestinal TJ protein structure and function by altering intracellular signaling, though these luminal factors may also directly or indirectly activate mucosal immune cells that locally release proinflammatory cytokines or mediators, that in turn may alter epithelial TJ structure and function. Although a change in diet alters the gut microbiota within $\sim 4$ days, which can recover within another $\sim 4$ days after the diet is normalized [9], the situation is more complex when a diet is chronically altered. Nevertheless, if a 15-day exposure to a high-fat diet universally alters duodenal and jejunal TJ structure as reported in the article [7], this suggests that the observed changes occur relatively rapidly. Further studies are expected to reveal the exact timing, segment and mechanisms, that will provide more information for key pathological factors, prevention of disease progression and therapeutic windows.

\section{References}

1. Hollander D, Kaunitz JD. The "Leaky Gut": Tight junctions but loose associations? Dig Dis Sci. 2020;65:1277-1287.

2. Cani PD, Bibiloni R, Knauf C, et al. Changes in gut microbiota control metabolic endotoxemia-induced inflammation in high-fat diet-induced obesity and diabetes in mice. Diabetes. 2008;57:1470-1481.

3. Weber CR. Dynamic properties of the tight junction barrier. Ann N Y Acad Sci. 2012;1257:77-84.

4. Camilleri M. Leaky gut: mechanisms, measurement and clinical implications in humans. Gut. 2019;68:1516-1526.

5. Akiba Y, Maruta K, Takajo T, et al. Lipopolysaccharides transport during fat absorption in rodent small intestine. Am J Physiol Gastrointest Liver Physiol. 2020;318:G1070-G1087.

6. Maruta K, Takajo T, Akiba Y, et al. GLP-2 acutely prevents endotoxin-related increased intestinal paracellular permeability in rats. Dig Dis Sci. 2020;65:2605-2618.

7. Nascimento JC, Matheus VA, Oliveira RB, Tada SFS, CollaresBuzato CB. High-fat diet induces disruption of the tight junction-mediated paracellular barrier in the proximal small intestine before the onset of type 2 diabetes and endotoxemia. Dig Dis Sci. (Epub ahead of print). https://doi.org/10.1007/s10620-020-06664 $-\mathrm{x}$.

8. de Oliveira RB, Matheus VA, Canuto LP, De Sant'ana A, Collares-Buzato CB. Time-dependent alteration to the tight junction structure of distal intestinal epithelia in type 2 prediabetic mice. Life Sci. 2019;238:116971.

9. David LA, Maurice CF, Carmody RN, et al. Diet rapidly and reproducibly alters the human gut microbiome. Nature. 2014;505:559-563.

Publisher's Note Springer Nature remains neutral with regard to jurisdictional claims in published maps and institutional affiliations. 